\title{
Effect of Microwave Sintering on Electrical Properties of Sr-deficient and Bi-rich Strontium Bismuth Niobate $\left(\mathrm{Sr}_{0.8} \mathrm{Bi}_{2.2} \mathrm{Nb}_{2} \mathrm{O}_{9}\right)$ Ferroelectric Ceramic
}

\author{
Rajveer Singh $^{1,2,3^{*}}$, Vandna Luthra ${ }^{3}$, R. P. Tandon ${ }^{1}$ \\ ${ }^{1}$ Department of Physics and Astrophysics, University of Delhi, Delhi-110007, India \\ ${ }^{2}$ Department of Physics, Atma Ram Santan Dharna College, University of Delhi, \\ Dhaula Kuan New Delhi-110021, India \\ ${ }^{3}$ Department of Physics, Gargi College, University of Delhi, Siri Fort road, New Delhi-110049, India \\ * Corresponding author email: rajveersingh@arsd.du.ac.in
}

Received: 27 July 2016 / Revised: 09 August 2016 / Accepted: 11 August 2016 / Published: 11 August 2016

\begin{abstract}
Nonstoichiometric strontium bismuth niobate $\left(\mathrm{Sr}_{0.8} \mathrm{Bi}_{2.2} \mathrm{Nb}_{2} \mathrm{O}_{9}\right.$ : $\left.\mathrm{SBN}\right)$ ceramic was prepared using conventional solid-state reaction and microwave sintering methods. Complex impedance spectroscopy (CIS) has been used to investigate the intra and intergranular contribution to the impedance SBN ceramics as a function of temperature and frequency. Complex impedance Cole-Cole plots were used to interpret the relaxation mechanism in SBN ceramic which showed a non-Debye relaxation. The grain and grain boundary contribution to conductivity have been estimated from the Cole-Cole plots. The bulk (grain) resistance of both samples was found to decrease with rise in temperature indicating negative temperature coefficient of resistance (NTCR) type behavior like that of semiconductors. The microwave sintered SBN was found to have low value of bulk resistance indicating more increase in conductivity as compared to conventionally sintered SBN. The temperature dependence of the relaxation time was found to obey the Arrhenius law. Studies of electrical modulus show the presence of hoping conduction mechanism in SBN.
\end{abstract}

Keywords: Bismuth layer Ferroelectric ceramics; Microwave sintering; Complex impedance and modulus spectroscopy

\section{Introduction}

The bismuth layered structure ferroelectric ceramics (BLSFs) have emerged as important candidates for the application in non-volatile ferroelectric random access memory (FeRAM) devices because of their attractive electrical properties such as high Curie temperature, low dielectric loss, excellent fatigue endurance and low aging rate [1-3]. The BLSFs ceramics belong to Aurivillious family having the chemical formula of $\left(\mathrm{Bi}_{2} \mathrm{O}_{2}\right)^{2+}\left(\mathrm{A}_{\mathrm{n}-1} \mathrm{~B}_{\mathrm{n}} \mathrm{O}_{3 \mathrm{n}+1}\right)^{2-}$, where $\mathrm{n}$ represents the number of perovskite layers sandwiched between two bismuth oxide layers.
The perovskite blocks $\left(A_{n-1} B_{n} O_{3 n+1}\right)^{2-}$ units are sandwiched between two layers of $\left(\mathrm{Bi}_{2} \mathrm{O}_{2}\right)^{2+}$ along the c-axis, where A-site in perovskite block can be occupied by cations having coordination no. of 12 such as $\mathrm{Bi}^{3+}, \mathrm{La}^{3+}, \mathrm{Sm}^{3+}, \mathrm{Ba}^{2+}, \mathrm{Ca}^{2+}, \mathrm{Sr}^{2+}$ etc. and $\mathrm{B}$ site, can be occupied by cations having coordination no. of 6 such as $\mathrm{Fe}^{3+}, \mathrm{Ti}^{3+}, \mathrm{Nb}^{5+}$, $\mathrm{Ta}^{5+}, \mathrm{W}^{6+}, \mathrm{Mo}^{6+}$, etc. [4-5]. Having the same structure, dielectric and ferroelectric properties of $\mathrm{SrBi}_{2} \mathrm{Nb}_{2} \mathrm{O}_{9}$ and other bismuth layer structured ferroelectrics (BLSF's) such as $\mathrm{SrBi}_{2} \mathrm{Ta}_{2} \mathrm{O}_{9}$, $\mathrm{SrBi}_{2} \mathrm{NbTaO}_{9}, \quad \mathrm{SrBi}_{4} \mathrm{Ta}_{4} \mathrm{O}_{15} ; \quad \mathrm{SrBi}_{2} \mathrm{Nb}_{2} \mathrm{O}_{9}$ has attracted much attention because of its high fatigue resistance and lead free composition [6-8]. 
In spite of the numerous advantages of SBN ceramics, it suffers from some serious issues such as relatively low remnant polarization, low piezoelectric coefficient and high processing temperature [7]. Numerous reports are available in the literature on the synthesis of strontium bismuth niobate and its solid solutions by processes such as solid-state reaction [6-10], aqueous solution method [11,12], organic precursor decomposition [13,14], coprecipitation $[15,16]$, sol-gel $[17,18]$, and combustion synthesis [19]. The conventional solid state reaction method is generally used for synthesizing SBN, which has some disadvantages such as high sintering temperature and loss of bismuth compound during the sintering process. The electrical properties of the SBN are strongly dependent on the density of ferroelectric ceramics [20]. Therefore, fully dense materials $(>95 \%$ of theoretical density) are required for most of the applications. The abnormal grain growth in ferroelectric ceramics is might be due to high sintering temperature and longer sintering durations. Higher sintering temperature not only leads to bismuth loss in SBN, but also affects its electrical properties. To overcome the issue of volatility of bismuth, available reports suggest the usage of microwave sintering. In the last two decades the microwave sintering has emerged for synthesizing ceramics at lower temperatures as compared to conventional sintering. The microwave sintering has many advantages over the conventional solid state reaction technique. It includes rapid heating, uniform grain growth, and higher densification [21, 22]. Several researchers have synthesized different material systems using this microwave technique. However, only few publications are available for the microwave sintering of BLSFs ceramics [23-27]. There is not much literature available on the effect of microwave sintering temperature on the electrical properties of SBN of ceramics. Therefore, in this paper, SBN ceramic was prepared by conventional solid state reaction and sintered by microwave reactive and conventional sintering techniques. The conventional sintering was used to aiming to compare the electrical properties by both techniques. Noguchi et al. [28] reported that SBN and SBT show the better dielectric and ferroelectric properties with the composition of Sr: $\mathrm{Bi}: \mathrm{Nb} / \mathrm{Ta}=0.8: 2.2: 2$ as compared to the composition of 1: 2: 2 [29-31]. In this study SBN was synthesized of the composition Sr: $\mathrm{Bi}: \mathrm{Nb}=$ 0.8:2.2:2 by conventional and microwave sintering techniques. The complex impedance spectroscopy is a powerful technique to separate out the grain-electrode effects and grain boundary effects. It provides important information about the contribution to the relaxation process from different micro-regions in polycrystalline ceramics, such as grains, grain boundaries and bulk-electrode interfaces [32, 33]. The present paper describes a detailed analysis of complex impedance spectroscopy and complex modulus spectroscopy for both the compounds synthesized by conventional and microwave sintering. An effort has been done to study the role of grain and grain boundaries on electrical properties of SBN synthesized by two different techniques and their dependence on frequency and temperature. Interestingly we have not come across hardly any publication related to complex impedance spectroscopy of SBN synthesized by this microwave technique during last five years.

\section{Experimental Details}

The high temperature solid state reaction conventional and microwave sintering techniques were used for the preparation of $\mathrm{Sr}_{0.8} \mathrm{Bi}_{2.2} \mathrm{Nb}_{2} \mathrm{O}_{9}$ (SBN) ceramic. SBN powder was prepared according to the equation

$$
\begin{aligned}
0.8\left(\mathrm{SrCO}_{3}\right)+ & \frac{2.2}{2}\left(\mathrm{Bi}_{2} \mathrm{O}_{3}\right)+\mathrm{Nb}_{2} \mathrm{O}_{5} \\
& \stackrel{\Delta}{\rightarrow} \mathrm{Sr}_{0.8} \mathrm{Bi}_{2.2} \mathrm{Nb}_{2} \mathrm{O}_{9}+\mathrm{CO}_{2} \uparrow
\end{aligned}
$$

The starting materials were $\mathrm{SrCO}_{3}$ (purity 99.99\%, Sigma Aldrich), $\mathrm{Bi}_{2} \mathrm{O}_{3}$ (purity 99.9\%, $\mathrm{CDH}$ ) and $\mathrm{Nb}_{2} \mathrm{O}_{5}$ (purity $99.99 \%$, Alfa Aesar). The powders were preheated at $100^{\circ} \mathrm{C}$ to remove the moisture and then weighed according to the composition of $\mathrm{Sr}_{0.8} \mathrm{Bi}_{2.2} \mathrm{Nb}_{2} \mathrm{O}_{9}$. Bismuth oxide $\left(\mathrm{Bi}_{2} \mathrm{O}_{3}\right)$ was used in $3 \%$ excess to compensate the loss of $\mathrm{Bi}$ during the sintering process. The powders were mixed for 24 hours in ball milling in isopropanol medium with Zirconia balls. The as-prepared powders were dried at $100^{\circ} \mathrm{C}$ and then calcined at $800^{\circ} \mathrm{C}$ for 4 hours in a closed alumina crucible using conventional furnace. The 
Singh et al., J. Mod. Mater.; Vol. 1, Issue 1, pp: 35-45, 2016

resultant powder was ground for two hours and then mixed with polyvinyl alcohol (PVA). The PVA mixed powder was pelletized into disk of 10 $\mathrm{mm}$ diameter and about $1.5 \mathrm{~mm}$ thickness using a uniaxial hydraulic pressure.

The one set of pellets of SBN were heated in conventional furnace at $1100^{\circ} \mathrm{C}$ for 3 hours with heating and cooling rate of $3^{\circ} \mathrm{C} /$ minutes. Another set of pellets of $\mathrm{SBN}$ were sintered at optimized temperature $1000^{\circ} \mathrm{C}$ for 30 minutes with a heating rate of $50^{\circ} \mathrm{C} / \mathrm{min}$ in a microwave furnace. A Micro-Heat High Performance Microwave furnace (model MH5814-VI, from Energy Microwave System Pvt. Ltd.) having two magnetrons with microwave power of 0.5 $\mathrm{KW}-1.45 \mathrm{KW}$ operated at $2.45 \mathrm{GHz}$ was used in the present study. The temperature inside the furnace was measured by an inferred fiber optic pyrometer. The pellets were loaded in a thermally insulating chamber size of $17 \mathrm{~cm}$ x $17 \mathrm{~cm}$ x $15 \mathrm{~cm}$ in size to prevent heat loss. The silicon carbide was used as microwave susceptor around the sample to preheat the sample within the insulating chamber. This configuration makes the microwave heating more uniform. The comparison of temperature-time profile for conventional sintering and microwave sintering is shown in figure 1.

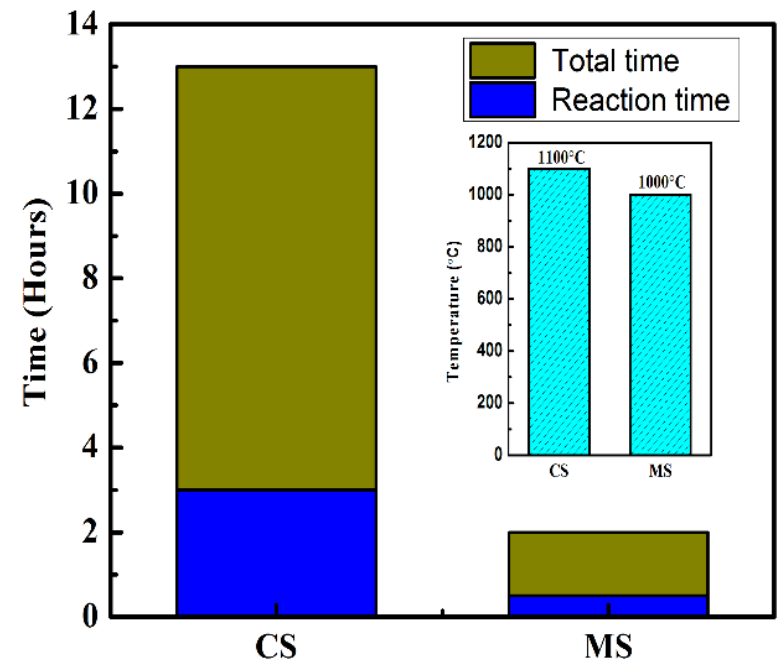

Figure 1: Comparison of Temperature-time profile for synthesis of SBN by conventional and microwave sintering.

It can be seen in the figure 1 that the time required for microwave sintering is $50 \mathrm{~min}$. only (excluding the cooling time) which is much lesser than for conventional sintering (approx. 9 hours excluding the cooling time). Therefore, the microwave sintering is beneficial to save energy and time. The SBN sintered by conventional and microwave sintering is abbreviated by CS-SBN and MS-SBN respectively in this study. Before electrical measurements both sintered pellets were lapped with silicon carbide powder to reduce thickness to about $1 \mathrm{~mm}$. The silver paste was used as electrode on both sides of pellets and fired at $500^{\circ} \mathrm{C}$ for 30 minutes. Electrical impedance $(Z)$, phase angle $(\theta)$, loss tangent $(D)$, and capacitance $(C)$ were measured as a function of frequency $(20 \mathrm{~Hz}-1 \mathrm{MHz})$ at different temperatures $\left(30-500^{\circ} \mathrm{C}\right)$ using computer interfaced Wayne Kerr 6500B Impedance analyzer at oscillation amplitude of $1 \mathrm{~V}$.

\section{Results and Discussion}

\subsection{Impedance Spectroscopy}

To investigate the electrical and dielectric properties of materials, complex impedance spectroscopy (CIS) is the well-known technique. It is used to characterize microstructural and electrical properties of ionic and / or electronic materials [34]. The grain, grain boundary and ceramics interface effects, greatly influence the electrical properties of ceramics materials. Therefore, it is required to understand the individual contribution to the electrical behavior. In general, four representations are used to represent data in complex plane, complex impedance $\left(Z^{*}\right)$, complex admittance $\left(Y^{*}\right)$, complex permittivity $\left(\varepsilon^{*}\right)$ and complex modulus $\left(M^{*}\right)$. Complex impedance plane plot of $Z^{\prime}$ versus $Z^{\prime \prime}$ (where $Z^{\prime}$ and $Z^{\prime \prime}$ are the real and imaginary parts of the complex impedance, respectively) are useful for determining the dominant resistance of a sample, while complex modulus plots are useful for determining the smallest capacitances. The advantage of this CIS technique is that the $M^{\prime \prime}$ and $Z^{\prime \prime}$ peaks for a particular RC combination should be dependable on the frequency scale which is the ideal Debye case of dielectric materials. The highlight of complex impedance is that it highlights the phenomena of largest resistance while, the complex modulus highlights the phenomena of smallest capacitance $[35,36]$. 

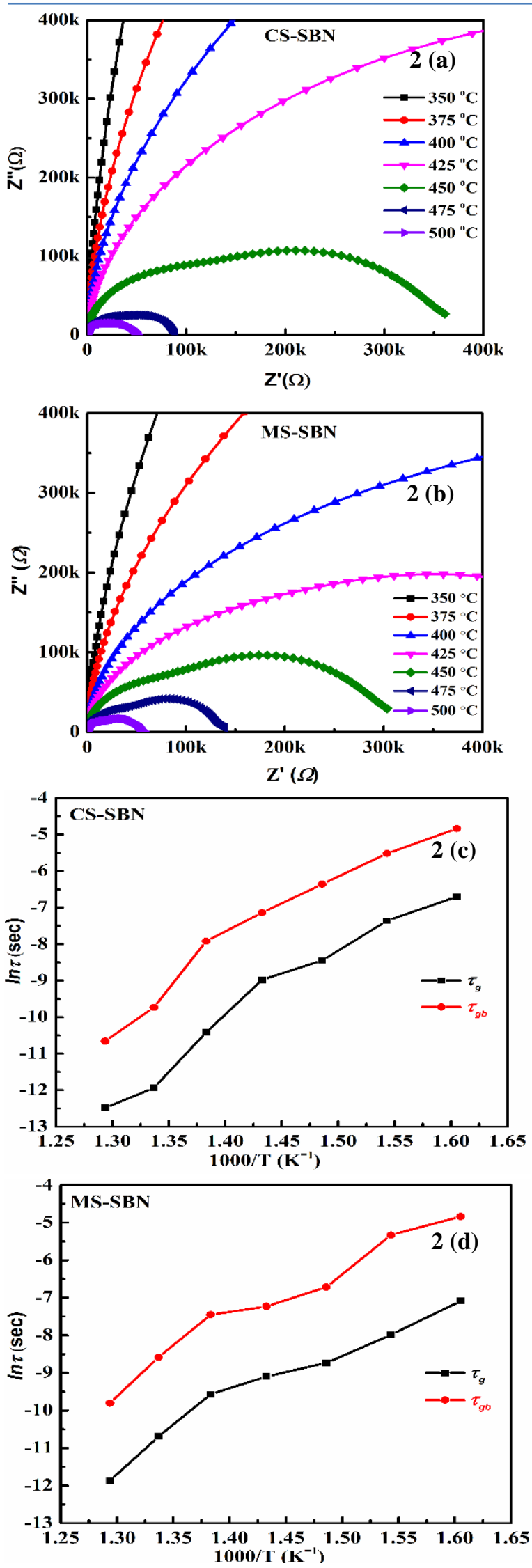

Figure 2: Complex impedance plots of (a) CS-SBN and (b) MS-SBN along with Arrhenius plots of relaxation time for grain and grain boundary for (c) . CS-SBN and (d) $M S-S B N$
Figure 2 shows the complex impedance plane plots of CS-SBN and MS-SBN samples measured at various temperatures from $350^{\circ} \mathrm{C}$ to $500^{\circ} \mathrm{C}$ in the frequency range $20 \mathrm{~Hz}$ to $1 \mathrm{MHz}$. At lower temperatures $\left(<400^{\circ} \mathrm{C}\right)$ the response of Cole-Cole plots were found linear for both samples CS-SBN and MS-SBN, which indicates the insulating behavior in the samples. This linear response of Cole-Cole plots have been found to change into semicircles above $400^{\circ} \mathrm{C}$ in both specimens indicating the increase in the conductivity of the specimens. Two semicircles were observed for both specimens CS-SBN and MS-SBN in low and high frequency regions respectively above $400^{\circ} \mathrm{C}$ with different values of resistances for grain and grain boundary. The non-appearance of third arc indicates that, the effect due to electrode is negligible. The complex plots were fitted using $\mathrm{Z}$ view software and the values of fitted parameters are listed in table 1.

Table1. Grain and grain boundary resistances for conventionally and microwave sintered SBN respectively.

\begin{tabular}{|c|c|c|c|c|}
\hline \multirow{2}{*}{$\begin{array}{l}\text { Tempe } \\
\text { rature } \\
\left({ }^{\circ} \mathrm{C}\right)\end{array}$} & \multicolumn{2}{|c|}{ CS-SBN } & \multicolumn{2}{|c|}{ MS-SBN } \\
\hline & $\begin{array}{l}R_{g} \\
(k \Omega)\end{array}$ & $\begin{array}{l}R_{\mathrm{gb}} \\
(\mathrm{k} \boldsymbol{\Omega})\end{array}$ & $\begin{array}{l}R_{g} \\
(k \Omega)\end{array}$ & $\begin{array}{l}R_{\mathrm{gb}} \\
(\mathrm{k} \Omega)\end{array}$ \\
\hline 400 & 1760.00 & 3200.00 & 587.00 & 863.00 \\
\hline 425 & 745.00 & 984.00 & 303.00 & 351.00 \\
\hline 450 & 167.00 & 251.00 & 130.00 & 144.00 \\
\hline 475 & 48.15 & 221.43 & 39.98 & 52.03 \\
\hline 500 & 29.40 & 120.66 & 17.63 & 16.13 \\
\hline
\end{tabular}

It can be seen from table 1 that grain and grain boundary resistance decreases with increase in temperature at all temperatures used in the measurements. The values of grain boundary resistances were observed higher than that of grain resistance for both samples CS-SBN and MS-SBN. Hence at higher temperatures, grain and grain boundary resistances could be separated which clearly explains the presence of grain boundaries in both samples CS-SBN and MS-SBN. The defects in BLSFs ceramics affects impedances and capacitance in the formation of barrier layer at the grain-grain boundary interface [37]. The defects are the oxygen vacancies which are usually created during sintering process. The barrier at the interfaces of grain-grain boundary is created due to the differences in the values of 
Singh et al., J. Mod. Mater.; Vol. 1, Issue 1, pp: 35-45, 2016

grain and grain boundary resistances. The decrease in value of bulk (grain) resistance with increase in temperature indicates the negative temperature coefficient of resistance (NTCR) behavior in both CS-SBN and MS-SBN ceramics. The relaxation time $(\tau)$ was calculated from the peak frequency of semicircles of complex impedance plots using the equation $\tau=1 /$ $2 \pi R C$ where, $R$ and $C$ are the resistance and capacitance for grain, grain boundary. Variation of grain and grain boundary relaxation time with temperature is shown in the figure 2 (c) and (d). It was observed that the relaxation time for grain boundary is more than that of grains. The relaxation times due to grain and grain boundary follow the Arrhenius behavior

$$
\tau=\tau_{0} \exp \left(-E_{\tau} / K T\right)
$$

where, $\tau_{0}$ is the pre-exponential factor. An anomalous behavior was observed in both plots near the transition temperature. The activation energy for grain $\left(E_{g}\right)$ and grain boundary $\left(E_{g b}\right)$ were estimated from linear least squares fit to $l n$ ( $\tau)-1000 / T$ and the values are given in table 2 .

Table 2. Activation energy for grain and grain boundary of CS-SBN and MS-SBN ceramics.

\begin{tabular}{|l|l|l|}
\hline $\begin{array}{l}\text { Sample } \\
\text { code }\end{array}$ & $\begin{array}{l}\text { Grain activation } \\
\text { energy }\left(\boldsymbol{E}_{\boldsymbol{g}}\right) \text { in } \\
\mathbf{e V}\end{array}$ & $\begin{array}{l}\text { Grain boundary } \\
\text { activation energy } \\
\left(\boldsymbol{E}_{\boldsymbol{g} b}\right) \text { in } \mathbf{e V}\end{array}$ \\
\hline $\mathrm{CS}-\mathrm{SBN}$ & 1.68 & 1.61 \\
$\mathrm{MS}-\mathrm{SBN}$ & 1.21 & 1.31 \\
\hline
\end{tabular}

The values of $E_{g}$ and $E_{g b}$ have been observed lower for MS-SBN than that of CS-SBN, indicating the higher conductivity in the samples at higher temperature. Figure 3 (a) and (b) show the variation of $Z^{\prime}$ with frequency at temperature between $350^{\circ} \mathrm{C}-500^{\circ} \mathrm{C}$ for CS-SBN and MS-SBN samples. It was observed that the value of $Z^{\prime}$ at low frequency is almost half for MS-SBN than that of CS-SBN. It means that microwave sintered SBN (MS-SBN) offers low resistance for charge carriers. The value of $Z^{\prime}$ decreases with both increasing frequency and temperature for both samples. The low frequency dispersion in $Z^{\prime}$ was observed below $1 \mathrm{kHz}$ frequency, and above $1 \mathrm{kHz}$, the $Z^{\prime}$ was found to be independent of frequency and temperature which is consistent with low frequency dielectric dispersion (LFDD).
This behavior may be due to the release of space charge carrier as a result of barrier potential with increase of temperature and it is also responsible for ac conductivity with temperature at high frequency.
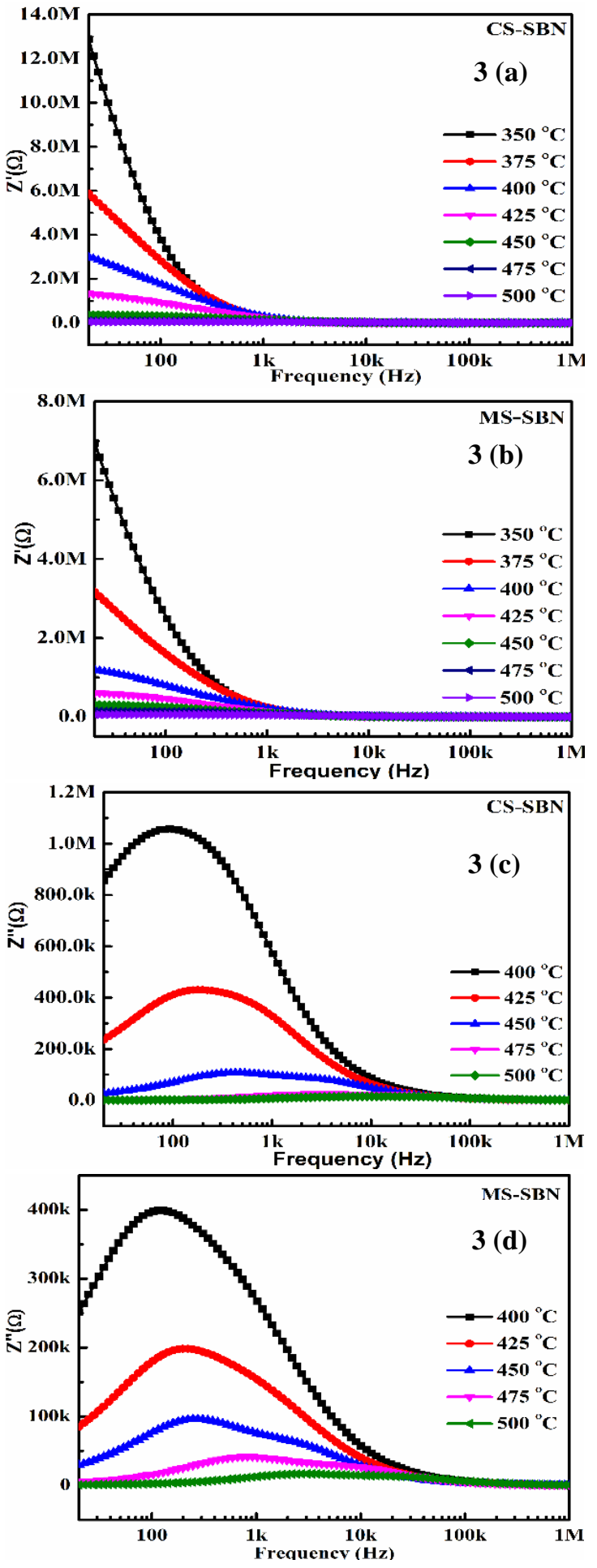

Figure 3: Frequency and temperature dependence of real part ( $\left.Z^{\prime}\right)(a)$, (b) and; (c) , (d) imaginary part ( $\left.Z^{\prime \prime}\right)$ of impedance for $C S-S B N$ and MS-SBN respectively. 
The decrease in $Z^{\prime}$ values at low frequency with increase in temperature indicate the negative temperature coefficients of resistance (NTCR) type behavior similar to semiconductor materials [38]. Figure 3 (c) and (d) show the variation of $Z^{\prime \prime}$ with frequency at different temperature for CS-SBN and MS-SBN samples. It was noticed that the $Z^{\prime \prime}$ values increases initially, attains a maxima peak and then decreases with increase in frequency for temperatures $\geq 400^{\circ} \mathrm{C}$. Such type of behavior belongs to non- Debye like behavior which indicates the presence of space charges since, the electrical behavior is dependent on the frequency. It was also observed that the peak value decreases with increase of temperature and shifted to the higher frequency side. This decrease in the value of $Z^{\prime \prime}{ }_{\text {max }}$ peak with increasing temperature represents the increase in capacitance and decrease in resistance of the material which indicates the increase in conductivity and loss in the resistive property of the material since $Z^{\prime \prime}{ }_{\text {max }}=R / 2$ [39]. The shifting in the peak value of $Z^{\prime \prime}{ }_{\text {max }}$ to the higher frequency side indicates that the relaxation time is more at low temperature and decrease with increase in temperature $[40,41]$. The broadening of the peaks was observed with increase in temperature which indicates multiple relaxations in the material. The microwave sintered SBN was found to have less broadening peaks. The relaxation may be due to the presence of immobile charge species at low temperature and defects at higher temperature [42]. At high frequency $(>10 \mathrm{kHz})$ all the curves were found to merge, which might be due to the presence of space charge in the material. At high frequencies, lesser time is available for the space charge to relax and hence the space charge polarization reduces with increasing frequency leading to merging.

The normalized imaginary parts $Z^{\prime \prime} / Z^{\prime \prime}{ }_{\max }$ of the impedance as a function of frequency at different temperatures is shown in the figure 4 for CS-SBN and MS-SBN ceramics. The Z"/Z" ${ }_{\max }$ parameter shows a peak at a particular frequency which shifts towards the higher frequency side with increase in temperature. It may be concluded from the curves that the higher temperature triggers another relaxation process in the material. It was also observed that the peaks tend to broadened with increasing temperature which indicates the presence of electrical processes in the material with a spread of relaxation time [37].
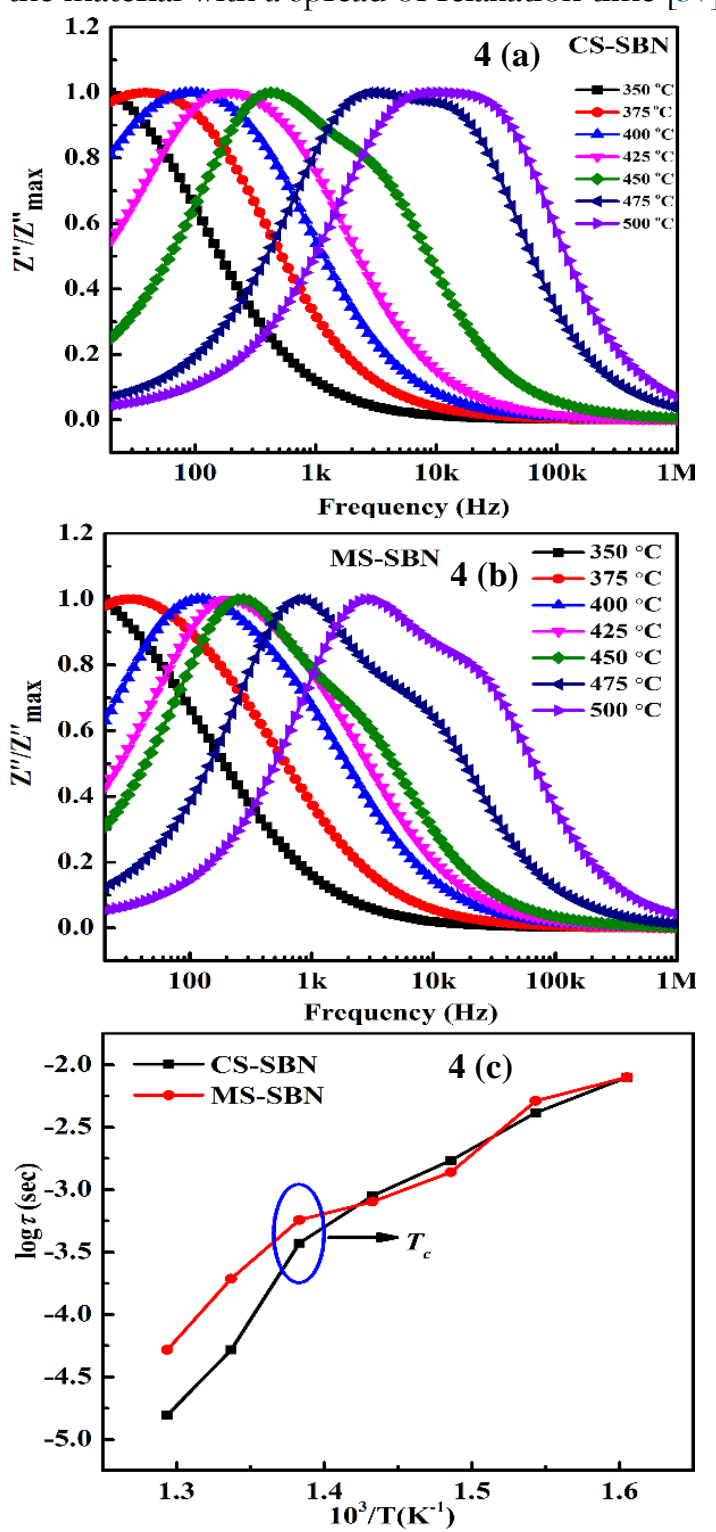

Figure 4: $(a)$ and $(b)$, the normalized imaginary parts $\left(Z^{\prime \prime} / Z^{\prime \prime}{ }_{\max }\right)$ of the impedances as a function of frequency at different temperatures and (c) the Relaxation time as a function of temperature for $C S-S B N$ and $M S-S B N$ respectively

The relaxation time $\left(\tau_{m}\right)$ was estimated using the relation

$$
2 \pi f_{m} \tau_{m}=1
$$

where, $\tau_{\mathrm{m}}$ is the relaxation time and $f_{m}$ is the peak frequency. Figure 4 (c) shows the Arrhenius plot of relaxation time with temperature. The relaxation time was found to decrease with increase in temperature. Both samples have the similar values of relaxation time and an anomalous behavior was observed near the transition temperature in case of both specimens. 
Singh et al., J. Mod. Mater.; Vol. 1, Issue 1, pp: 35-45, 2016

The activation energies in ferroelectric region $\left(350-450^{\circ} \mathrm{C}\right)$ were estimated from the Arrhenius plots for CS-SBN and MS-SBN, they are $1.18 \mathrm{eV}$ and $1.12 \mathrm{eV}$ respectively which correspond to the oxygen migration energy. The activation energy for oxygen vacancy migration is about $1.00 \mathrm{eV}$ [43]. Since Bi ions evaporated at high sintering temperatures, oxygen vacancies are created for neutralization in the crystal structure [44]. Hence the bismuth vacancies and oxygen vacancies are considered to be the most mobile charges which are responsible for electrical conduction in SBN.

\subsection{Electrical Modulus Study}

Complex modulus spectroscopy is also a very important tool to understand the charge transport process in ionic solids. It provides an alternative approach to analyze the electrical response of the materials. Electrical modulus was introduced by Macedo et al. [45] to study the space charge relaxation phenomenon. The complex modulus spectroscopy also helps to understand the uncertainty arising in the relation with the presence of grain/grain boundary effect at elevated temperature which may not be distinguished from the complex impedance plots [46]. The complex modulus electrical modulus $\left(M^{*}\right)$ was calculated from the impedance data using the relation,

$$
\begin{gathered}
M^{*}(\omega)=1 / \varepsilon^{*}(\omega)=i \omega C_{0} Z^{*}(\omega) \\
=M^{\prime}+i M^{\prime \prime} \\
M^{\prime}=\omega C_{0} Z^{\prime \prime} \\
M^{\prime \prime}=\omega C_{0} Z^{\prime}
\end{gathered}
$$

where, $\varepsilon^{*}(\omega)$ is the complex permittivity, $C_{0}$ is the geometrical capacitance $\left(\varepsilon_{0} A / t, A\right.$ be the electrode area and $\mathrm{t}$ be the thickness of the sample. Figure 5 (a) and (b) show the variation of real part of electrical modulus with frequency for CS-SBN and MS-SBN samples in the temperature range $350^{\circ} \mathrm{C}$ to $500^{\circ} \mathrm{C}$. It was noticed that $M^{\prime}$ increases with increase in frequency from zero and attained constant value at higher frequencies above $1 \mathrm{kHz}$ which is $M_{\infty}$. It can be attributed to the conduction phenomena arising from short range mobility of charge carriers. It was also noticed that the value of $M_{\infty}$ decreases with increase in temperature and attains a minimum value below the transition temperature $\left(T_{c}\right)$ and above $T_{c}$ it again increases. The low frequency dispersion region was observed which shift to the higher frequency side with increase in temperature. The trend of the curves was found different beyond the transition temperature in both samples (circled in the plots). Above the transition temperature, the value of $M^{\prime}$ was found approx. zero up to $5 \mathrm{kHz}$ and then increases in case of both specimens.
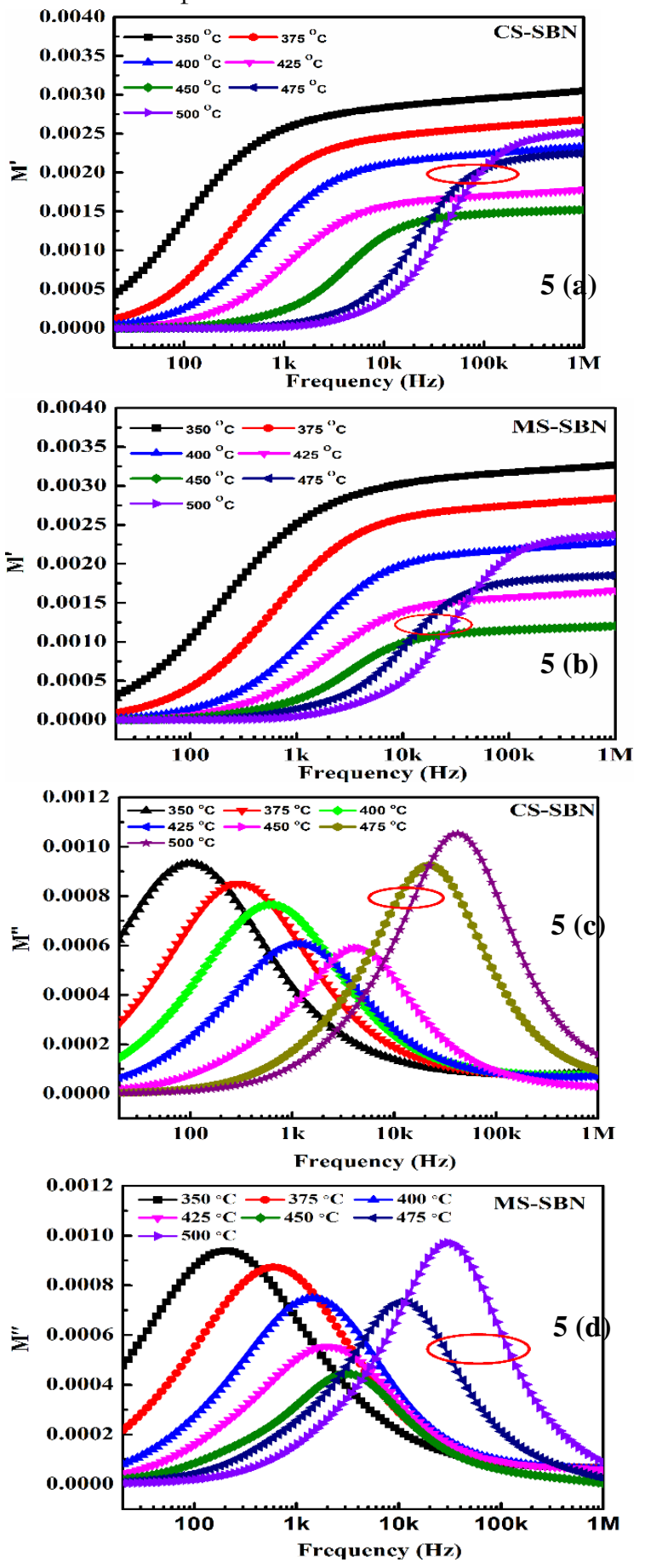

Figure 5: Frequency dependence of real part $\left(M^{\prime}\right)$ (a), (b) and; (c), (d) imaginary part $\left(M^{\prime \prime}\right)$ of electrical modulus for $C S-S B N$ and $M S-S B N$ respectively. 
Figure 5 (c) and (d) show the variation of imaginary part of electrical modulus $\left(M^{\prime \prime}\right)$ with frequency for CS-SBN and MS-SBN at different temperatures. The $M^{\prime \prime}$ was found to increase with increase in frequency and attains a peak at a particular frequency $\left(f_{p}\right)$, which represents the most provable conductivity relaxation frequency. The peak height of $M^{\prime \prime}$ was found to decrease and shifted to the higher frequency side with increase in temperature and attains a minimum value corresponding to transition temperature which is a direct indication of increase in the value of dielectric constant. The value of peak height increases with increase in temperature beyond the transition temperature and shifted to higher frequency side. However the values of $M^{\prime \prime}$ for MS-SBN were slightly lower than that of CS-SBN as the dielectric constant for MS-SBN is higher. This indicates that the dielectric relaxation is a thermally activated process in which the hopping mechanism dominates intrinsically [41]. These plots highlight the phenomenon of smallest capacitance because the peak height is proportional to $1 / C$ i.e. $M_{\max }=\varepsilon_{0} / 2 C$ [41]. The peaks of $M^{\prime \prime}$ indicate the transition from short range to long range mobility with decrease in frequency. The low frequency side is the nonDebye peaks which represent the range of frequencies in which the ions are capable of moving long distance, i.e., performing successful hopping from one site to a neighboring site, whereas for higher frequency side, the ions are spatially confined to their potential wells and can execute only localized motion [39]. Above the transition temperature, $M^{\prime \prime}$ almost start from zero and increases with increase in frequency and then attains a peak value which again shifted to higher frequency side with further increase in temperature (circled in the plots). The further increase in the magnitude of $M^{\prime \prime}$ with increase in temperature above transition temperature indicates that the capacitance is decreased with increase in temperature. Figure 6 (a) and (b) show the normalized imaginary parts of electrical modulus $M^{\prime \prime} / M^{\prime \prime}{ }_{\text {max }}$ as a function of frequency for CS-SBN and MS-SBN at different temperatures. The peak frequency of $M^{\prime \prime} / M^{\prime \prime}$ max has been found to shift towards the higher frequency region which may be related to the phenomenon that at higher temperature there is another relaxation process.
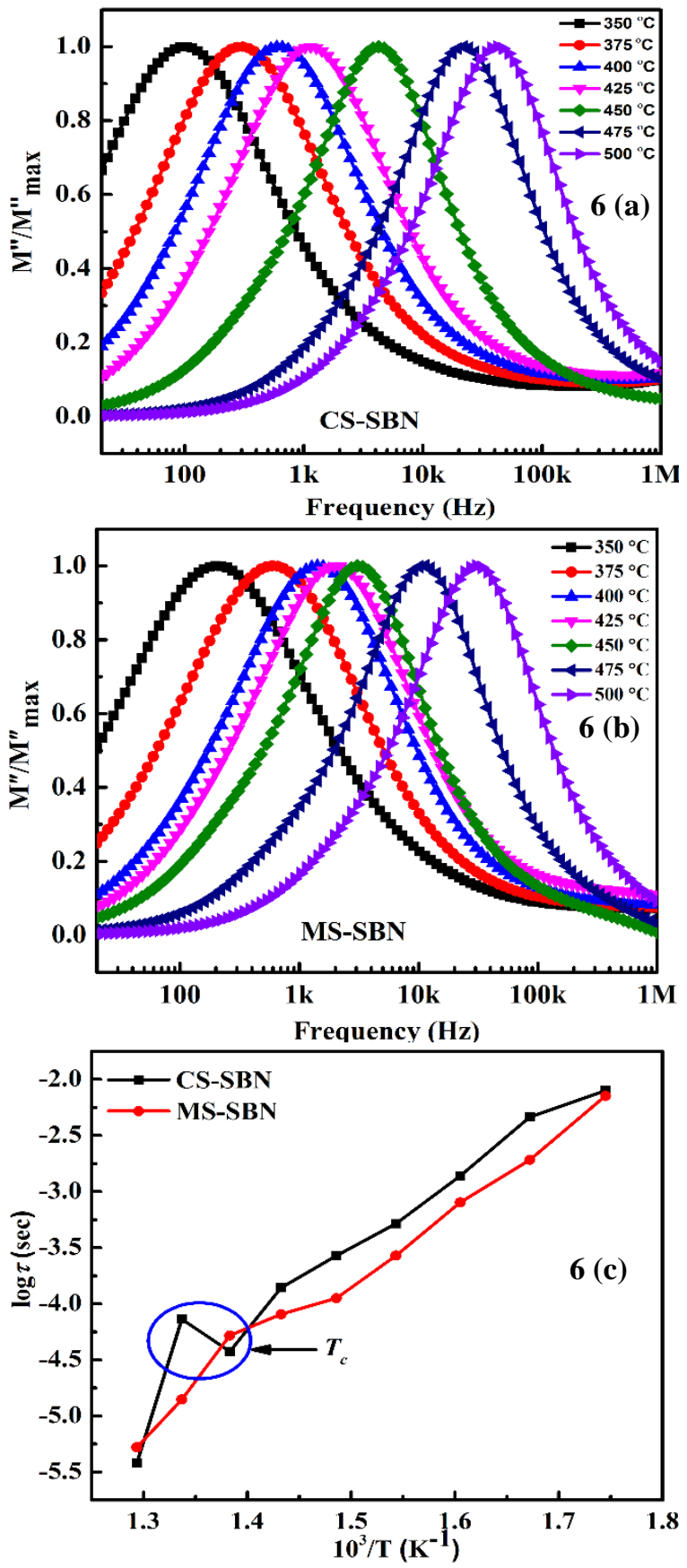

Figure 6: $(a)$ and $(b)$, the normalized imaginary parts $\left(M^{\prime \prime} / M^{\prime \prime}{ }_{\max }\right)$ of the impedances as a function of frequency at different temperatures and (c) the Relaxation time as a function of temperature for $C S-S B N$ and $M S-S B N$ respectively.

The relaxation time $\left(\tau_{m}\right)$ was estimated from the peak frequency $f_{m}$ in $M^{\prime \prime}$ using the relation

$$
2 \pi f_{m} \tau_{m}=1
$$

The relaxation time $\left(\tau_{m}\right)$ has been observed to follow Arrhenius law $\tau_{m}=\tau_{0} \exp \left(-E_{a} / K T\right)$ shown in figure 6 (c), where, $\tau_{0}$ is the pre- 
exponential factor, $K$ the Boltzmann constant, $E_{a}$ is the activation energy and $T$ is the absolute temperature. The value of relaxation time was found to decrease with increase in temperature. An anomalous was observed near the transition temperature for both samples. The activation energy has been calculated in the ferroelectric region (below $T_{c}$ ) they are $1.34 \mathrm{eV}$ and $1.29 \mathrm{eV}$ for CS-SBN and MS-SBN respectively. The activation energies estimated from $Z^{\prime \prime}$ and $M^{\prime \prime}$ plots were found similar which indicates that the localized conduction (dielectric relaxation) of the charge carriers in the bulk of the sample.

The variation of normalized parameters $M^{\prime \prime} / M^{\prime \prime}{ }_{\text {max }}$ and $Z^{\prime \prime} / Z^{\prime \prime}{ }_{\text {max }}$ as a function of frequency measured at $475^{\circ} \mathrm{C}$ is shown in the figure 7 (a) and (b) for CS-SBN and MS-SBN. A significant mismatch in the peaks of $M^{\prime \prime}$ and $Z^{\prime \prime}$
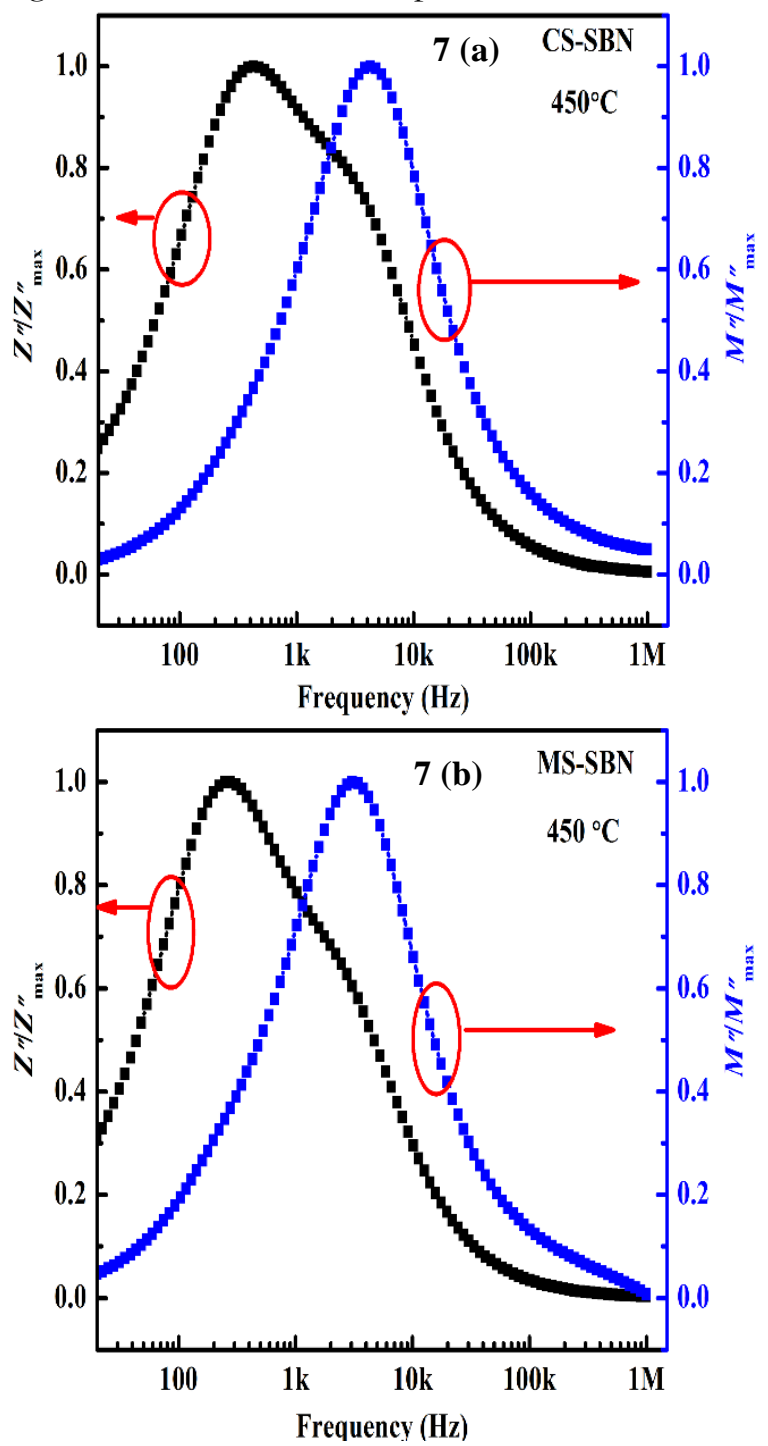

Figure 7: Normalized curves of $Z^{\prime \prime}$ and $M^{\prime \prime}$ for (a) $C S-S B N$ and (b) $M S-S B N$. has been observed. The mismatch in the peak position of normalized parameters $M^{\prime \prime}$ and $Z^{\prime \prime}$ indicates the presence of Maxwell-Wagner type space charge polarization which arises at defects (grain boundaries, sample electrode interfaces) presents in the sample [47]. These plots also show the non-Debye type behavior. In ideal case the $M^{\prime \prime}$ and $Z^{\prime \prime}$ should coincident on the frequency scale. The peak frequency in $M^{\prime \prime} / M^{\prime \prime}{ }_{\max }$ was found to be shifted more high frequency side as in $Z^{\prime \prime} / Z^{\prime \prime}{ }_{\max }$.

\section{Conclusion}

In summary, $\mathrm{Sr}_{0.8} \mathrm{Bi}_{2.2} \mathrm{Nb}_{2} \mathrm{O}_{9}$ compound was synthesized by both conventional and microwave sintering processes. The conventional reactive sintering was used with an objective to compare the electrical properties of SBN. The frequency and temperature dependent of complex impedance and modulus of conventional and microwave sintered SBN was studied. The bulk and grain boundary resistances were estimated from the Cole-Cole plots of impedance for both samples CS-SBN and MS-SBN which indicates that the relaxation is of non-Debye type. The lower value of grain boundary suggest that the conduction is dominated by grain boundary through hopping electrons created due to oxygen vacancies at higher temperature. The microwave sintered SBN was found to have low value of grain and grain boundary resistance at higher temperatures indicating low resistance for charge carriers for hopping from one site to another site. The frequency dependence of complex modulus $\left(M^{\prime \prime}\right)$ with temperature showed a maxima peak which shift towards the higher frequency side with increase in temperature. The shifting in the peak value of $M^{\prime \prime}$ indicates that the relaxation in the material is thermally activated. Thus it may be concluded that the microwave sintering technique is superior to conventional sintering technique to enhance the electrical properties of SBN from the point of view time saving, energy saving and low processing sintering temperature.

\section{Acknowledgment}

One of the authors (Rajveer Singh) is very thankful to Principal ARSD College, University of Delhi for granting study leave and Principal 
Gargi College for their support and help. The Authors R. Singh and V. Luthra thanks to Star College Grant (Department of Biotechnology) to Gargi College wide SAN/ No. 102/IFD/DBT/SAN/1911/2008-09.

\section{How to Cite this Article:}

R. Singh, V. Luthra, and R. Tandon, "Effect of Microwave Sintering on Electrical Properties of Sr-deficient and Bi-rich Strontium Bismuth Niobate $\left(\mathrm{Sr}_{0.8} \mathrm{Bi}_{2.2} \mathrm{Nb}_{2} \mathrm{O}_{9}\right)$ Ferroelectric Ceramic", J. Mod. Mater., vol. 1, no. 1, pp. 35-45, Aug. 2016. doi:10.21467/jmm.1.1.35-45

\section{References}

[1] De Araujo, C. A. P., Cuchiaro, J. D., McMillan, L. D., Scott, M. C. \& Scott, J. F., "Fatigue-free ferroelectric capacitors with platinum electrodes", Nature., V. 374, pp 627-629, 1995.

[2] Yao, Zhongran, et al. "Preparation and electrical properties of MoO 3-modified $\mathrm{SrBi} 2 \mathrm{Nb} 2 \mathrm{O}$ 9-based lead-free piezoelectric ceramics." Journal of Alloys and Compounds, V. 666, pp. 10-14, 2016.

[3] Yao, Zhongran, et al. "Preparation and electrical properties of $\mathrm{SrBi} 2-$ x $\mathrm{Sm}$ x $\mathrm{Nb2O}$ 9 lead-free piezoelectric ceramics." Journal of Materials Science: Materials in Electronics, V.27, No.2, pp2114-2119.B, 2016.

[4] B. Aurivillius, Mixed bismuth oxides with layer lattices. I. Structure type of $\mathrm{CaCb}_{2} \mathrm{Bi}_{2} \mathrm{O}_{9}$, Ark Kemi 1 , pp.463-480, 1949.

[5] B. H. Park, B. S. Kang, S.D. Bu, T. W. Noh, J. Lee, W. Jo, "Lanthanum-substituted bismuth titanate for use in non-volatile memories", Nature, V. 401, pp. 682-684, 1999.

[6] T. Wei, C. Z. Zhao, Q. J. Zhou, Z. P. Li, Y. Q. Wang, L.s Zhang, Bright green up-conversion emission and enhanced ferroelectric polarization in $\mathrm{Sr}_{1-1.5 \mathrm{x}} \mathrm{Er}_{\mathrm{x}} \mathrm{Bi}_{2} \mathrm{Nb}_{2} \mathrm{O}$ 9, Optical. Mater. V. 36, pp. 1209-1212, 2014.

[7] Z. Yao, R. Chu, Z. Xu, J. Hao, D. Wei, G. Li, "Dielectric, ferroelectric and piezoelectric properties of $\mathrm{Ca}_{0.1} \mathrm{Sr}_{0.9} \mathrm{Bi}_{2} \mathrm{Nb}_{2} \mathrm{O}_{9}$ ceramic", J. Mater. Sci.: Mater. Electron. V. 26, pp.8740-8746, 2015.

[8] I. Coondoo, A. K. Jha, S. K. Agarwal, "Enhancement of dielectric characteristics in donor doped Aurivillius $\mathrm{SrBi}_{2} \mathrm{Ta}_{2} \mathrm{O}_{9}$ ferroelectric ceramics", J. Eur. Ceram. Soc. V. 27, pp. 253-260, 2007.

[9] D. Kajewski, Z. Ujma, K. Szot, M. Pawełczyk, "Dielectric properties and phase transition in $\mathrm{SrBi}_{2} \mathrm{Nb}_{2} \mathrm{O}_{9}-\mathrm{SrBi}_{2} \mathrm{Ta}_{2} \mathrm{O}_{9}$ solid solution", Ceram. Int. V. 35 pp. 2351-2355, 2009.

[10] W. Wu, S. Liang, X. Wang, J. Bi, P. Liu, L. Wu "Synthesis, structures and photo catalytic activities of microcrystalline $\mathrm{ABi}_{2} \mathrm{Nb}_{2} \mathrm{O}_{9}(\mathrm{~A}=\mathrm{Sr}, \mathrm{Ba})$ powders", $J$. Solid State Chem. V. 184, pp.81-88, 2011.

[11] D. Dhak, P. Dhak, P. Pramanik, "Influence of substitution on dielectric and impedance spectroscopy of $\mathrm{Sr}_{1-\mathrm{x}} \mathrm{Bi}_{2+\mathrm{y}} \mathrm{Nb}_{2} \mathrm{O}_{9}$ ferroelectric ceramics synthesized by chemical route", Appl. Surf. Sci. V. 254, pp. 3078-3092, 2008.

[12] D. Nelis, D. Mondelaers, G. Vanhoyland, A. Hardy, K. Van Werde, H. V. den Rul, M. K. Van Bael, J. Mullens, 1. C. Van Pocke, J. D. Haen, "Synthesis of strontium bismuth niobate $\left(\mathrm{SrBi}_{2} \mathrm{Nb}_{2} \mathrm{O}_{9}\right)$ using an aqueous acetate- citrate precursor gel: thermal decomposition and phase formation", Thermochemica. Acta. V. 426, pp.39-48, 2005.

[13] D. Prasanta, D. Debasis, P. Kausikisankar, P. Panchanan, "Studies of structural and electrical properties of $\mathrm{Ca}_{1-\mathrm{x}} \mathrm{Bi}_{2+\mathrm{y}} \mathrm{Nb}_{2} \mathrm{O}_{9} \quad[0.0 \leq \mathrm{x} \leq 0.4 ; 0.000 \leq \mathrm{y} \leq 0.266]$ ferroelectric ceramics prepared by organic precursor decomposition method", Solid State Sci. V. 10 , pp.1936-1946, 2008.

[14] N. L. A Junior, A. Z. Simoes, A. A. Cavalheiro, S. M. Zanetti, E. Longo, J. A. Varela "Structural and microstructural characterization of $\mathrm{SrBi}_{2}\left(\mathrm{Ta}_{0.5} \mathrm{Nb}_{0.48} \mathrm{~W}_{0.02}\right)_{2} \mathrm{O}_{9}$ powders". J Alloy. Compd. $V$. 454, pp. 61-65, 2008.

[15] S. P. Gaikwad, S. R. Dhage, H. S. Potdar, V. Samuel, V. Ravi, "Co-precipitation method for the preparation of nanocrystalline ferroelectric $\mathrm{SrBi}_{2} \mathrm{Nb}_{2} \mathrm{O}_{9}$ ceramics", $J$. Electroceram. V. 14, pp. 83-87, 2005.

[16] S. P. Gaikwad, H. S. Potdar, V. Samuel, V. Ravi, "Coprecipitation method for the preparation of fine ferroelectric $\mathrm{BaBi}_{2} \mathrm{Nb}_{2} \mathrm{O}_{9}$," Ceram. Int. V. 31 pp. 379-381, 2005.

[17] K. Kato, C. Zheng, J. M. Finder, S. K. Dey, Y. Torii, "Sol-gel route to ferroelectric layer-structured perovskite $\mathrm{SrBi}_{2} \mathrm{Ta}_{2} \mathrm{O}_{9}$ and $\mathrm{SrBi}_{2} \mathrm{Nb}_{2} \mathrm{O}_{9}$ thin films", $J$. Am. Ceram. Soc. V. 81, pp.1869-1875, 1998.

[18] D. Nelis, K. V. Werde, D. Mondelaers, G. Vanhoyland, H. V. D. Rul, M. K. V. Bael, J. Mullens, L. C. V. Poucke, "Aqueous solution-gel synthesis of strontium bismuth niobate $\left(\mathrm{SrBi}_{2} \mathrm{Nb}_{2} \mathrm{O}_{9}\right)$ ", J. Sol Gel Sci. Technol. V. 26, pp.1125-1129, 2003.

[19] S. M. Zanetti, E. I. Santiago, L. O. S. Bulhoes, J. A. Varela, E. R. Leite, E. Longo, "Preparation and characterization of nanosized $\mathrm{SrBi}_{2} \mathrm{Nb}_{2} \mathrm{O}_{9}$ powder by the combustion synthesis", Materials Letters V. 57, pp. 2812-2816, 2003.

[20] A. Kumar, S. R. Emani, V. V. B. Prasad, K. C. J. Raju, A. R. James, "Microwave sintering of fine grained PLZT $8 / 60 / 40$ ceramics prepared via high energy mechanical milling", J. Eur. Ceram. Soc. V. 36, no. 10, pp. 2505-2511, 2016.

[21] X. H. Zhu, Q. M. Hang, "Microscopical and physical characterization of microwave and microwavehydrothermal synthesis products", Micron. V. 44, pp. 21-44, 2013.

[22] D. Agarwal, "Microwave sintering of ceramics, composites and metal powders, In: Z. Z. Fang Ed. Sintering of advanced materials", UK: Woodhead Publishing; pp. 222-248, 2010.

[23] N. Azurmendi, I. Karo, A. C. Caballero, T. Jardiel T, M. Villegas, "Microwave-Assisted Sintering of Bismuth Titanate-Based Ceramics", J. Am. Ceram. Soc. V. 80, pp.1232-1236, 2006.

[24] S. Kumar, M. Panneerselvam, K. J. Rao, "Microwave Synthesis and Sintering of $\mathrm{Bi}_{4} \mathrm{Ti}_{3} \mathrm{O}_{12}$, the Aurivillius Compound: Structural and Chemical Effects of attempted Lithiation", Ferroelectr. V. 306, pp.165-177, 2004.

[25] Sugandha, A. K. Jha, "Synthesis and characterization of nanocrystalline ferroelectric $\mathrm{Sr}_{0.8} \mathrm{Bi}_{2.2} \mathrm{Ta}_{2} \mathrm{O}_{9}$ by conventional and microwave sintering: A comparative study", Mater. Res. Bull. V. 48, pp. 1553-1559, 2013.

[26] S. N. Kumar, P. Kumar, D. K. Agrawal, "Structural, dielectric and ferroelectric properties of SBN ceramics synthesized by microwave reactive sintering technique", Ceram. Int. V. 38, pp5243-5250, 2012. 
Singh et al., J. Mod. Mater.; Vol. 1, Issue 1, pp: 35-45, 2016

[27] A. Dias A, R. L. Moreira, "Production of Sr-deficient bismuth tantalates from microwave hydrothermal derived precursors: Structural and dielectric properties", J. Phys. Chem. Solids. V. 68, pp. 645-649, 2007.

[28] T. Noguchi, T. Hase, Y. Miyasaka, "Analysis of the Dependence of Ferroelectric Properties of Strontium Bismuth Tantalate (SBT) Thin Films on the Composition and Process Temperature", Jpn. J. Appl. Phys. V. 35,pp 4900-4904, 1996.

[29] T. Atsuki, N. Soyama, T. Yonezawa, K. Ogi, "Preparation of Bi-Based Ferroelectric Thin Films by Sol-Gel Method", Jpn. J. Appl. Phys. V. 34, pp. 5096-5099, 1995.

[30] H. M. Tsai, P. Lin, T.Y. Tseng, " $\mathrm{Sr}_{0.8} \mathrm{Bi}_{2.5} \mathrm{Ta}_{1.2} \mathrm{Nb}_{0.9} \mathrm{O}_{9+\mathrm{x}}$ ferroelectric thin films prepared by two-target off-axis radio frequency magnetron sputtering", Appl. Phys. Lett. V. 72, pp.1787-1789, 1998.

[31] R. Singh, V. Luthra, R. S. Rawat, R. P. Tandon, "Structural, dielectric and piezoelectric properties of $\mathrm{SrBi}_{2} \mathrm{Nb}_{2} \mathrm{O}_{9}$ and $\mathrm{Sr}_{0.8} \mathrm{Bi}_{2.2} \mathrm{Nb}_{2} \mathrm{O}_{9}$ ceramics", Ceram. Int. $\mathrm{v}$. 41, pp.4468-4478, 2015.

[32] Y. Wu, M. J. Forbess, S. Seraji, S. J. Limmer, T. Chou, G. Z. Cao, "Impedance study of $\mathrm{SrBi}_{2} \mathrm{Ta}_{2} \mathrm{O}_{9}$ and $\mathrm{SrBi}_{2}\left(\mathrm{Ta}_{0.9} \mathrm{~V}_{0.1}\right)_{2} \mathrm{O}_{9}$ ferroelectrics", Mater. Sci. Eng. $B$ v. 86, pp 70-78, 2001.

[33] T. C. Chen, C. L. Thio, S. B. Desu, "Impedance spectroscopy of $\mathrm{SrBi}_{2} \mathrm{Ta}_{2} \mathrm{O}_{9}$ and $\mathrm{SrBi}_{2} \mathrm{Nb}_{2} \mathrm{O}_{9}$ ceramics correlation with fatigue behavior", J. Mater. Res., v. 12, pp. 2628-2637, 1997.

[34] J. R. Macdonald, "Impedance spectroscopy", Wiley, New York, 1987.

[35] K. S. Rao, D. M. Prasad, P. M. Krishna, B. Tilak, K. Ch. Varadarajulu, "Impedance and modulus spectroscopy studies on $\mathrm{Ba}_{0.1} \mathrm{Sr}_{0.81} \mathrm{La}_{0.06} \mathrm{Bi}_{2} \mathrm{Nb}_{2} \mathrm{O}_{9}$ ceramic", Mater. Sci. Eng. B, v. 133, pp.141-150, 2006.

[36] K. S. Rao, D. M. Prasad, P. M. Krishna, B. H. Bindu, K. Suneetha, "Frequency and temperature dependence of electrical properties of barium and gadolinium substituted $\mathrm{SrBi}_{2} \mathrm{Nb}_{2} \mathrm{O}_{9}$ ceramics", J. Mater. Sci. v. 42, pp.7363-7374, 2007.

[37] C. K. Suman, K. Prasad, R. N. P. Choudhary, "Complex impedance studies on tungsten-bronze electroceramic: $\mathrm{Pb}_{2} \mathrm{Bi}_{3} \mathrm{LaTi}_{5} \mathrm{O}_{18}$," J. Mater. Sci., v. 41, pp.369-375, 2006.

[38] K. S. Cole, R. H. Cole, "Dispersion and Absorption in Dielectrics I. Alternating Current Characteristics", $J$. Chem. Phys., v. 9 , pp341-351, 1941.

[39] I. Coondoo, N. Panwar, A. Tomar, A.K. Jha, S.K. Agarwal, "Impedance spectroscopy and conductivity studies in $\operatorname{SrBi}_{2}\left(\mathrm{Ta}_{1-\mathrm{x}} \mathrm{W}_{\mathrm{x}}\right)_{2} \mathrm{O}_{9}$ ferroelectric ceramics", Phys. B , v.407, pp. 4712-4720, 2012.

[40] D. C. Sinclair, A. R. West, "Impedance and modulus spectroscopy of semiconducting $\mathrm{BaTiO}_{3}$ showing positive temperature coefficient of resistance", J. Appl. Phys., v. 66, pp. 3850-3856, 1989.

[41] B. Behera, P. Nayak, R. N. P. Choudhary, "Structural and impedance properties of $\mathrm{KBa}_{2} \mathrm{~V}_{5} \mathrm{O}_{15}$ ceramics", Mater. Res. Bull., v. 43, pp. 401-410, 2008.

[42] E. Barsoukov, J. R. M., ed. "Impedance Spectroscopy", John-Willey: New York. Pp. 305-314, 2005.

[43] J. S. Kim, B. C. Choi, H. K. Yang, J. H. Jeong, S.T. Chung, S. B. Cho, "Low Frequency Dielectric Dispersion and Electrical Conductivity of Pure and LaDoped $\mathrm{SrBi}_{2} \mathrm{Nb}_{2} \mathrm{O}_{9}$ Ceramics", J. Korean Phys. Soc. V.52, pp. 415-420, 2008.

[44] I. W. Kim, C. W. Ahn, J. S. Kim, T. K. Song, J. S. Bae, B. C. Choi, J. H. Jeong, J. S. Lee, "Low-frequency dielectric relaxation and ac conduction of $\mathrm{SrBi}_{2} \mathrm{Ta}_{2} \mathrm{O}_{9}$ thin film grown by pulsed laser deposition", Appl. Phys. Lett. V. 80, pp. 4006, 2002.

[45] P. B. Macedo Macedo, C. T. Moynihan, R. Boseat, "Role of ionic diffusion in polarization in vitreous ionic conductors", Phys. Chem. Glasses, v. 13, pp.171-179, 1992.

[46] R. N. P. Choudhary, D. K. Pradhan, C. M. Tirado, G. E. Bonilla, R. S. Katiyar, "Impedance characteristics of $\mathrm{Pb}\left(\mathrm{Fe}_{2 / 3} \mathrm{~W}_{1 / 3}\right) \mathrm{O}_{3}-\mathrm{BiFeO}_{3}$ composites", Phys. Status Solidi B, v. 244, pp.2254-2266, 2007.

[47] R. Gerhardt, "Impedance and Dielectric Spectroscopy Revisited: Distinguishing Localized Relaxation from Long-Range Conductivity" J. Phys. Chem. Solids, V. 55 pp. 1491-1506, 1994.

Publish your research article in AIJR journals-

$\checkmark$ Online Submission and Tracking

$\checkmark$ Peer-Reviewed

$\checkmark$ Rapid decision

$\checkmark$ Immediate Publication after acceptance

$\checkmark$ Articles freely available online

$\checkmark \quad$ Retain full copyright of your article.

Submit your article at journals.aijr.in

Publish your books with AIJR publisher-

$\checkmark$ Publish with ISBN and DOI.

$\checkmark$ Publish Thesis/Dissertation as Monograph.

$\checkmark$ Publish Book Monograph.

$\checkmark$ Publish Edited Volume/ Book.

$\checkmark$ Publish Conference Proceedings

$\checkmark$ Retain full copyright of your books.

Submit your manuscript at books.aijr.org 